\title{
Paper
}

\section{An automated design procedure for class-E amplifier}

\author{
Yuichi Tanji ${ }^{1 a)}$, Haruna Matsushita ${ }^{1 b)}$, and Hiroo Sekiya ${ }^{2 c)}$ \\ ${ }^{1}$ Faculty of Engineering and Design, Kagawa University \\ 2217-20 Hayashi-cho, \\ Takamatsu, Kagawa 761-0396, Japan \\ ${ }^{2}$ Graduate School of Engineering, Chiba University, \\ Chiba, 263-8522, Japan \\ a) tanji.yuichi@kagawa-u.ac.jp \\ b)haruna@ieee.org \\ c) sekiya@faculty.chiba-u.ac.jp
}

Received January 31, 2020; Revised April 28, 2020; Published October 1, 2020

\begin{abstract}
Class-E amplifier is one of the switching amplifiers. It is difficult to determine the circuit parameters satisfying the switching conditions. Although the Newton method was proposed to determine the passive elements, it requires a good initial solution that is obtained by using an analytical expression of the class-E amplifier. Even if the circuit configuration is slightly changed, the analytical expression must be re-derived. In this paper, the passive element value determination of class-E amplifier is defined as an optimization problem. This problem is solved by some methods which do not rely on the initial solution obtained by using the analytical expression. Furthermore, the objective function value for the optimization is efficiently calculated via a behavioral model of the class-E amplifier, which eases the optimum circuit design computationally and economically. Thus, an automated design procedure for the class-E amplifier will be presented in this paper.
\end{abstract}

Key Words: simplex method, Newton-Raphson method, Resonant Power Converters

\section{Introduction}

The class-E switching-mode circuits are used as valuable components in many applications, e.g., radio transmitters, switching mode-dc power supplies, devices of medical applications, and so on [1]. Because of the class-E switching, both zero voltage switching (ZVS) and zero derivative switching (ZDS), the class-E switching circuits can achieve high power-conversion efficiency at high frequencies. However, the class-E amplifier design is complicated because the two switching conditions must be satisfied simultaneously on the steady-state waveform.

The analytical expressions are very useful for the class-E amplifier design. Many analytical descriptions for the class-E amplifier design have been presented [2-8]. Early designs assumed an ideal switch, infinite output network Q (i.e., sinusoidal output current), and an RF choke in the dc supply 
lead (i.e., constant current drive) [2,3]. Later work allowed finite output Q [4,5], drain-current fall time [6], and nonlinear parasitic capacitance on the active device $[7,8]$. Although these analytical expressions are very useful, we cannot guarantee that the class-E amplifier satisfies the class-E ZVS and ZDS conditions rigorously.

In $[1,9]$, an optimization procedure for the class-E amplifier design is proposed using Newton method. In this procedure, the design problem of the class-E amplifier is defined as solving a set of nonlinear equations that constrains the passive element values including in the circuit and the classE ZVS and ZDS conditions. The Newton method, more precisely, the Newton-Raphson method is applied to find the solution. Newton-Raphson method provides an accurate solution, but it requires a good initial solution to converge. Thus, the designers have to calculate the initial solution using the above analytical expressions of the class-E amplifier. Even if the design is slightly changed, the analytical expression must be reproduced; thus, this procedure using the Newton-Raphson method is not fully automated. Alternatively, a black-box optimization procedure is presented in [11], in which the ZVS and ZDS conditions are calculated by a commercial circuit simulator. Then, particle swarm optimization (PSO), which is a metaheuristic algorithm [10], is applied to find the solution. To evaluate the class-E ZVS and ZDS conditions, the steady-state response must be calculated. Unfortunately, the class-E amplifier is a high output Q circuit, which makes the transition time very long. Thus, the steady-state analysis of the class-E amplifier is very costly, even if a simulator which directly calculates the steady-state response is used. As a result, the optimization procedure using PSO requires approximately half a day in order to determine only two parameters. Even worse, the class-E amplifier design is not complete by determining a set of design parameters once. To know information such as power-conversion efficiency, distortion, and so on, many design candidates must be provided using the optimization. Although the class-E amplifier is a small-scale circuit, the design thus is computationally very expensive. Therefore, if one intends to execute it reasonably, many computational resources are required, which is economically expensive.

In this paper, an improved optimization procedure for the class-E amplifier design is presented. This is a fully automated design procedure that does not depend on a good initial guess for the optimization. Thus, the analytical expressions of the class-E amplifier are not required. To reduce the computational cost for the optimization, a behavioral model of the class-E amplifier is provided. Then, an efficient steady-state analysis can be done, and the ZVS and ZDS conditions of the class$\mathrm{E}$ amplifier are efficiently evaluated. Next, the passive element values determination of the class-E amplifier is defined as an optimization problem through preliminary optimization tests. Here, we show that it is difficult to design the class-E amplifier by using ZVS and ZDS conditions only, i.e., these conditions precisely correspond to the class-E frequency multipliers. An objective function, which constrains the frequency of output waveform, is introduced in order to prevent to converge into the solutions which correspond to the class-E frequency multipliers. After applying a grid search to the problem with the objective function that constrains the frequency, the simplex method [20] and the Newton-Raphson one are applied to find the global minimum point that satisfies the class-E ZVS and ZDS conditions. The grid point whose objective function value is the smallest within all grid points is used as an initial solution for these methods. We also show that the simplex method with an initial solution, which is near the origin in the parameter space, finds the global minimum point more efficiently than the methods with the grid search. In [13], Independent-minded PSO (IPSO), which is an enhanced version of PSO [12], is used for finding the design parameters of the class-E amplifier. As IPSO is a metaheuristic algorithm, we may expect that IPSO is not necessarily faster than the proposed methods, but it provides a better solution. Surprisingly, however, it will be shown in the numerical examples that the proposed procedure is more reliable than the IPSO-based design one.

To provide many candidates of the class-E amplifier, we must calculate the design parameters repeatedly changing the design specification gradually. Then, to find a series of the solutions efficiently, a curve tracing algorithm using Newton-Raphson method is proposed. The proposed optimization procedure neither requires many computational resources nor relies on the analytical expressions that designers with expert knowledge of the class-E amplifier can only derive. Although the mathematical and computational expertise is necessary to develop the design automation tool, once developed, 
designers can use this tool to design the class-E amplifier. This procedure can be also extended to other resonant power amplifier designs [16].

This paper is organized as follows. In Section 2, an efficient steady-state analysis of the class-E amplifier and the problem definition for the passive element values determination are presented. In Section 3, a curve tracing algorithm is presented in order to provide many sets of design parameters efficiently, in which the simplex method and the Newton-Raphson one with/without grid search are provided, which are a component of the curve tracing algorithm. In Section 4, some examples, which illustrate the effectiveness of the optimization algorithms, and design examples are presented. Finally, conclusions are drawn in Section 6.

\section{Optimization of class-E amplifier}

\subsection{Circuit topology and principle operation}

Figure 1(a) shows a circuit topology of the class-E amplifier. The class-E amplifier consists of dcsupply voltage source $V_{D}$, dc-feed inductor $L_{C}, n$-channel MOSFET $S$ as a switching device, shunt capacitor $C_{S}$, and a series resonant circuit $L_{0}-C_{0}-R$. Figure 2 shows example steady-state waveforms of the class-E amplifier. The MOSFET turns on and off by the driving signal $D_{r}$. When the switch is in the on-state, the voltage across the MOSFET is zero and the current flows through the MOSFET. While the switch is in the off-state, the difference of the currents through the dc-feed inductor and resonant network flows through the shunt capacitor, which generates a pulse-shape voltage waveform as shown in Fig. 2. In the nominal operation of the class-E amplifier, the switch turns on when both the switch voltage and its derivative are zero simultaneously on the steady-state. These switching conditions are referred to as the class-E ZVS and ZDS conditions, which are respectively expressed as

$$
v_{s}\left(\theta_{\text {on }}\right)=0 \text {, }
$$

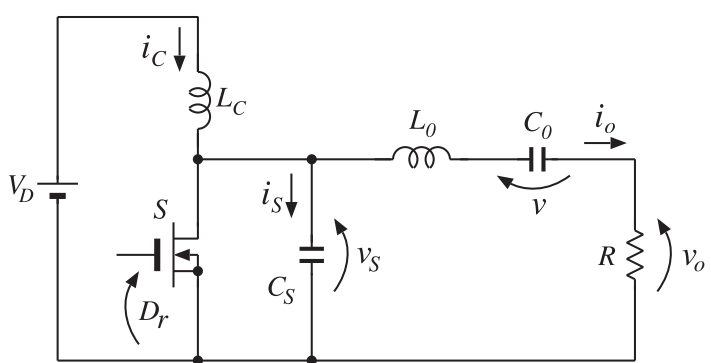

(a)

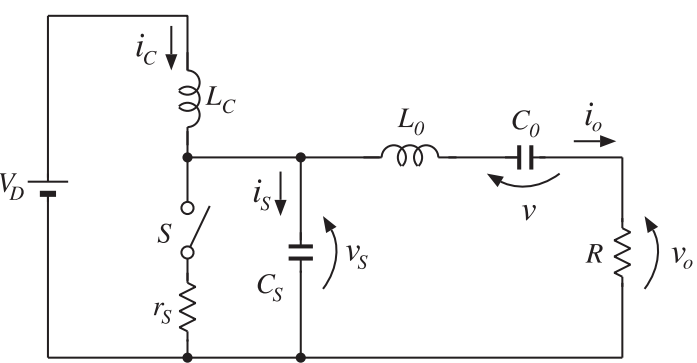

(b)

Fig. 1. A class E amplifier, (a) circuit model with MOSFET and (b) circuit model with an ideal switch.
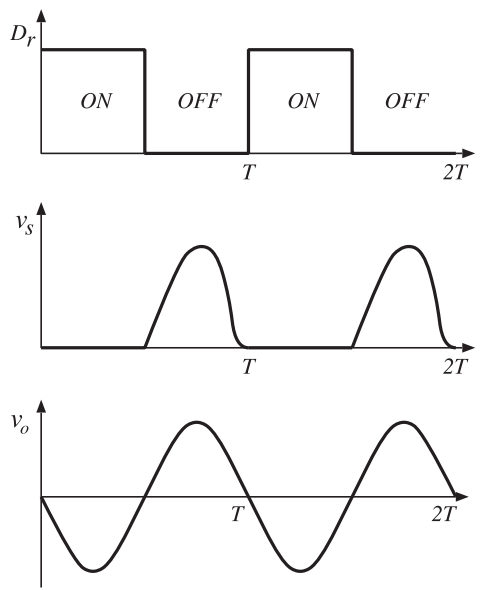

Fig. 2. Typical steady-state waveforms of class-E amplifier. 


$$
\left.\frac{d v_{s}}{d \theta}\right|_{\theta=\theta_{\mathrm{on}}}=0
$$

where $\theta=\omega t=2 \pi f t$ is the angular time and $f$ is the operating (switching) frequency. Additionally, $\theta_{\text {on }}$ is the angular time at the switch turn-on instant. By satisfying the class-E ZVS and ZDS conditions, switching losses are reduced and high power-conversion efficiency at high frequencies can be achieved. The passive elements should be determined uniquely to satisfy the class-E ZVS and ZDS conditions, which is not an easy task because the two conditions need to be filled simultaneously on the steady-state.

\subsection{Circuit equations and waveform expressions}

The resonant filter of the class-E amplifier usually has a high output $\mathrm{Q}$ value. Therefore, it takes a long transition time for the class-E amplifier to reach the steady-state. As the class-E ZVS and/or ZDS conditions are defined on the steady-state operation, it is not easy to find the passive element values achieving the class-E ZVS and ZDS conditions, using the transient analysis. With circuit simulators such as SPICE, we can carry out transient analysis, in which sophisticated MOSFET models are available. Then, the optimization to find the passive element values using SPICE is a black-box optimization, but it suffers from its heavy computational cost. Even though circuit simulators, which have a tool that steady-state waveforms can be obtained efficiently, are used, the computation is still costly for the optimization [11]. Therefore, the MOSFET in the circuit of Fig. 1(a) is modeled as an ideal switch as shown in Fig. 1(b). By using the ideal switching model, the circuit equations are expressed by piece-wise linear differential equations. Therefore, the solutions can be derived explicitly and we use them for obtaining the steady-state waveforms.

In this paper, the design parameters of the class-E amplifier are given as follows [9]:

1) $\omega=2 \pi f$ : the operating (switching) angular frequency,

2) $\omega_{0}=2 \pi f_{0}=1 / \sqrt{L_{0} C_{0}}$ : the resonant angular frequency,

3) $Q=\omega L_{0} / R$ : the loaded quality factor,

4) $A=f_{0} / f=\omega_{0} / \omega$ : the ratio of the resonant frequency to the operating frequency,

5) $B=C_{0} / C_{S}$ : the ratio of the capacitance of the resonant circuit capacitor to that of the shunt capacitor,

6) $H=L_{0} / L_{C}$ : the ratio of the inductance of the resonant circuit inductor to that of the dc-feed inductor, and

7) $D$ : the switch-off duty ratio of the switch, in which $0<D<1$.

Additionally, we assume that

a) the switching device works as an ideal switch; zero on-resistance, infinite off-resistance, and zero switching time,

b) shunt capacitance $C_{S}$ includes MOSFET parasitic capacitances,

c) all the passive elements including parasitic capacitors of MOSFET are linear and have no parasitic resistance, and

d) the switch turns on and off at $\theta=2 \pi$ and $\theta=2 \pi D$, respectively.

By applying these parameters and putting assumptions, the circuit equations can be formulated by 


$$
\begin{aligned}
& \left\{\begin{aligned}
\frac{d}{d \theta} \frac{R i_{C}}{V_{D}} & =\frac{H}{Q} \\
\frac{v_{S}}{V_{D}} & =0 \\
\frac{d}{d \theta} \frac{R i_{o}}{V_{D}} & =-\frac{1}{Q}\left(\frac{R i_{o}}{V_{D}}+\frac{v}{V_{D}}\right) \\
\frac{d}{d \theta} \frac{v}{V_{D}} & =A^{2} Q \frac{R i_{o}}{V_{D}}
\end{aligned}\right. \\
& \left\{\begin{aligned}
\frac{d}{d \theta} \frac{R i_{C}}{V_{D}} & =\frac{H}{Q}\left(1-\frac{v_{S}}{V_{D}}\right) \\
\frac{d}{d \theta} \frac{v_{S}}{V_{D}} & =A^{2} B Q\left(\frac{R i_{C}}{V_{D}}-\frac{R i_{o}}{V_{D}}\right) \\
\frac{d}{d \theta} \frac{R i_{o}}{V_{D}} & =\frac{1}{Q}\left(\frac{v_{S}}{V_{D}}-\frac{R i_{o}}{V_{D}}-\frac{v}{V_{D}}\right) \\
\frac{d}{d \theta} \frac{v}{V_{D}} & =A^{2} Q \frac{R i_{o}}{V_{D}}
\end{aligned} \quad \text { for } 0 \leq \theta \leq 2 \pi D\right.
\end{aligned}
$$

In the above equations, $(Q, H, D)$ is given as a design specification and $\boldsymbol{X}=[A, B]^{T}$ is set as the design parameters. Note that the circuit parameters, $V_{D}, R$, and $f$, are given a priori. If $\boldsymbol{X}$ is determined, all the passive elements are determined; thus, the problem to find $\boldsymbol{X}$ is referred to as the passive element values determination of the class-E amplifier.

The system with (3) and (4) is a linear-time-invariant system. Thus, the solutions of (3) and (4) are expressed via the eigenvalue decomposition of the matrices

$$
\boldsymbol{\alpha}_{1}=\left[\begin{array}{cc}
-\frac{1}{Q} & -\frac{1}{Q} \\
A^{2} Q & 0
\end{array}\right]
$$

for $(3)^{1}$ and

$$
\boldsymbol{\alpha}_{2}=\left[\begin{array}{cccc}
0 & -\frac{H}{Q} & 0 & 0 \\
A^{2} B Q & 0 & -A^{2} B Q & 0 \\
0 & \frac{1}{Q} & -\frac{1}{Q} & -\frac{1}{Q} \\
0 & 0 & A^{2} Q & 0
\end{array}\right]
$$

for (4), respectively. By using the eigenvalues and eigenvectors, the solutions $\boldsymbol{x}(\theta)=\left[x_{1}(\theta), x_{2}(\theta)\right.$, $\left.x_{3}(\theta), x_{4}(\theta)\right]^{T}=\left[R i_{C} / V_{D}, v_{S} / V_{D}, R i_{o} / V_{D}, v / V_{D}\right]^{T}$ of (3) and (4) are obtained as

$$
\begin{aligned}
& \boldsymbol{x}_{\mathrm{on}}(\theta)=\gamma_{1}(\theta) \boldsymbol{x}\left(0_{-}\right)+\boldsymbol{\phi}_{1}(\theta), \\
& \boldsymbol{x}_{\mathrm{off}}(\theta)=\gamma_{2}(\theta) \boldsymbol{x}(2 \pi D)+\phi_{2}(\theta),
\end{aligned}
$$

where

$$
\begin{aligned}
\gamma_{1}(\theta) & =\left[\begin{array}{ccc}
1 & 0 & 0 \\
0 & 0 & 0 \\
0 & \tilde{\boldsymbol{\gamma}}_{1}(\theta)
\end{array}\right], \\
\boldsymbol{\phi}_{1}(\theta) & =\left[\begin{array}{cccc}
\frac{H}{Q} \theta & 0 & 0 & 0
\end{array}\right]^{T}, \\
\tilde{\boldsymbol{\gamma}}_{1}(\theta) & =\boldsymbol{S}_{1} \operatorname{diag}\left\{e^{\lambda_{1,1} \theta}, e^{\lambda_{2,1} \theta}\right\} \boldsymbol{S}_{1}^{-1}, \\
\boldsymbol{\gamma}_{2}(\theta) & =\boldsymbol{S}_{2} \operatorname{diag}\left\{e^{\lambda_{1,2} \theta}, \cdots, e^{\lambda_{4,2} \theta}\right\} \boldsymbol{S}_{2}^{-1}, \\
\boldsymbol{\phi}_{2}(\theta) & =\boldsymbol{S}_{2} \operatorname{diag}\left\{\frac{e^{\lambda_{1,2} \theta}-1}{\lambda_{1,2}}, \ldots, \frac{e^{\lambda_{4,2} \theta}-1}{\lambda_{4,2}}\right\} \boldsymbol{S}_{2}^{-1}\left[\begin{array}{cccc}
\frac{H}{Q} & 0 & 0 & 0
\end{array}\right]^{T} .
\end{aligned}
$$

${ }^{1}$ The solutions of the first and second equations of $(3)$ are $(H / Q) \theta$ and 0 , respectively. 
Here, $\lambda_{1,1}, \lambda_{2,1}$ and $\lambda_{1,2}, \cdots, \lambda_{4,2}$ are respectively eigenvalues of $\boldsymbol{\alpha}_{1}$ and $\boldsymbol{\alpha}_{2}$, and $\boldsymbol{S}_{1}$ and $\boldsymbol{S}_{2}$ are matrices whose columns are the corresponding eigenvectors. Note that the continuity at $\theta=0$ is not guaranteed, because (3) is a descriptor system rather than a set of ordinary differential equations ${ }^{2}$. Thus, the solution $\boldsymbol{x}_{\text {on }}(\theta)$ depends on $\boldsymbol{x}\left(0_{-}\right)$[15].

As (4) is a set of ordinary differential equations, the solution's continuity holds at $\theta=2 \pi D$. Using (5), the condition is expressed as $\boldsymbol{x}(2 \pi D)=\boldsymbol{x}_{\text {on }}(2 \pi D)=\gamma_{1}(2 \pi D) \boldsymbol{x}\left(0_{-}\right)+\boldsymbol{\phi}_{1}(2 \pi D)$. On the other hand, the steady-state condition is provided as $\boldsymbol{x}\left(0_{-}\right)=\boldsymbol{x}_{\mathrm{off}}(2 \pi)=\boldsymbol{\gamma}_{2}(2 \pi) \boldsymbol{x}(2 \pi D)+\boldsymbol{\phi}_{2}(2 \pi)$. Combining the two conditions, we can obtain the initial values as

$$
\boldsymbol{x}\left(0_{-}\right)=\left(\boldsymbol{I}-\boldsymbol{\gamma}_{2}(2 \pi) \boldsymbol{\gamma}_{1}(2 \pi D)\right)^{-1}\left(\boldsymbol{\gamma}_{2}(2 \pi) \boldsymbol{\phi}_{1}(2 \pi D)+\boldsymbol{\phi}_{2}(2 \pi)\right),
$$

where $\boldsymbol{I}$ is an identity matrix. The steady-state response of the class-E amplifier can be calculated by using (5) with the initial values of (6).

The class-E ZVS condition (1) is divided by $V_{D}$. Then, the ZVS condition is evaluated at $\theta_{\text {on }}=0_{-}$ as

$$
x_{2}\left(0_{-}\right)=0 .
$$

The ZDS condition (1) is multiplied by $C_{S} R / V_{D}$. Then, the relationship, $C_{S} R / V_{D} d v_{S} / d t=$ $R / V_{D}\left(i_{C}(\theta)-i_{o}(\theta)\right)$, holds in the off-state. Thus, the class-E ZDS condition is evaluated as

$$
x_{1}\left(0_{-}\right)-x_{3}\left(0_{-}\right)=0 .
$$

The behavioral modeling in this subsection is based on the state space modeling [14]. We extend this method to the case where the idealized circuit in the on-state is expressed by a descriptor system. When the idealized circuit has a more complicated structure, more generalized modeling can be used [15]. Therefore, the proposed optimization method can be applied to other types of resonant power amplifier designs [16].

\subsection{Problem definition}

In this subsection, we define the problem of the passive element values determination for the class-E amplifier. Before providing the definition, preliminary tests were performed. To achieve the ZVS and/or ZDS conditions of (7) and (8), the tested objective functions were defined as

$$
\begin{aligned}
& f_{1}(A, B)=\left(x_{2}\left(0_{-}\right)\right)^{2}, \\
& f_{2}(A, B)=\left(x_{1}\left(0_{-}\right)-x_{3}\left(0_{-}\right)\right)^{2}, \\
& f_{3}(A, B)=\left(x_{2}\left(0_{-}\right)\right)^{2}+\left(x_{1}\left(0_{-}\right)-x_{3}\left(0_{-}\right)\right)^{2},
\end{aligned}
$$

where $f_{1}(A, B), f_{2}(A, B)$, and $f_{3}(A, B)$ were used to find the global minimum points that satisfy the ZVS, ZDS, and both ZVS and ZDS conditions, respectively. In the dynamic equations of (3) and (4), a design specification $(Q, H, D)$ is fixed and the design parameters $(A, B)$ are changed for the optimization. Then, the initial values $\boldsymbol{x}\left(0_{-}\right)$of $(6)$, which provide a steady-state response, depend on $(A, B)$. This implies that $(A, B)$ changes the objective function values of $f_{1}(A, B), f_{2}(A, B)$, and $f_{3}(A, B)$, implicitly. If a minimization algorithm is applied to each problem associated with $f_{1}(A, B)$, $f_{2}(A, B)$, and $f_{3}(A, B)$, respectively, the passive element values of the class-E amplifiers that satisfy the ZVS, ZDS, and both ZVS and ZDS conditions, respectively, can be found.

Preliminary optimization tests were run, in which the class-E amplifier expressed by (3) and (4) was analyzed, set to $Q=30, H=0.001$, and $D=0.5$. As an algorithm for the minimization, PSO was applied to find the global minimum points, restricted as $A, B \in[0.1,2.0]$. In Appendix, PSO is explained in a part of IPSO, which is an extended version of PSO, where the relationship between a particle's position of PSO and the design parameters of the class-E amplifier is expressed as $\boldsymbol{X} \equiv\left(x_{1}, x_{2}\right) \equiv(A, B)$. For PSO, the parameters were set as Table I in which the values of $M, w$,

\footnotetext{
${ }^{2}$ The second line of (3) is not a differential equation. Then, (3) is called a descriptor system, the solution's continuity of which is not guaranteed, differently from ordinary differential equation cases.
} 
Table I. Parameters used in the methods in this paper.

\begin{tabular}{|c|c|c|c|}
\hline methods & content & symbols & value \\
\hline $\mathrm{PSO} / \mathrm{IPSO}$ & $\begin{array}{l}\text { population size } \\
\text { inertia weight } \\
\text { acceleration coefficients } \\
\text { maximum number of iterations } \\
\text { termination tolerance on function value of (11) }\end{array}$ & $\begin{array}{c}M \\
w \\
c_{1}, c_{2} \\
N_{0} \\
\Theta_{1}\end{array}$ & $\begin{array}{c}24 \\
0.729 \\
1.494 \\
5,000 \\
10^{-10}\end{array}$ \\
\hline $\begin{array}{c}\text { PSO/IPSO } \\
\text { SMPLX-G1/SMPLX-G2/NEW-G2 }\end{array}$ & $\begin{array}{l}\text { lower limit of searching interval } \\
\text { upper limit of searching interval }\end{array}$ & $\begin{array}{l}x_{\min } \\
x_{\max }\end{array}$ & \\
\hline IPSO & cooperative coefficient & $K$ & 0.1 \\
\hline SMPLX-G1/SMPLX-G2/NEW-G2 & division number of searching interval & $N_{1}$ & \\
\hline SMPLX-G1/SMPLX-G2/SMPLX & $\begin{array}{l}\text { maximum number of iterations } \\
\text { maximum number of function evaluations } \\
\text { termination tolerance on function value of (11) } \\
\text { termination tolerance on solution variation }\end{array}$ & $\begin{array}{l}N_{0} \\
N_{2} \\
\Theta_{1} \\
\Theta_{2}\end{array}$ & $\begin{array}{c}5,000 \\
10,000 \\
10^{-10} \\
10^{-10}\end{array}$ \\
\hline SMPLX & a small positive number in (15) & $\epsilon_{1}$ & \\
\hline NEW-G2/Curve Tracing & $\begin{array}{l}\text { a small positive number in }(20) \\
\text { termination tolerance on function value of (11) } \\
\text { maximum number of linear search iterations } \\
\text { maximum number of Newton-Raphson } \\
\text { iterations }\end{array}$ & $\begin{array}{l}\epsilon_{2} \\
\Theta_{1} \\
N_{3} \\
N_{4}\end{array}$ & $\begin{array}{c}10^{-10} \\
10\end{array}$ \\
\hline
\end{tabular}

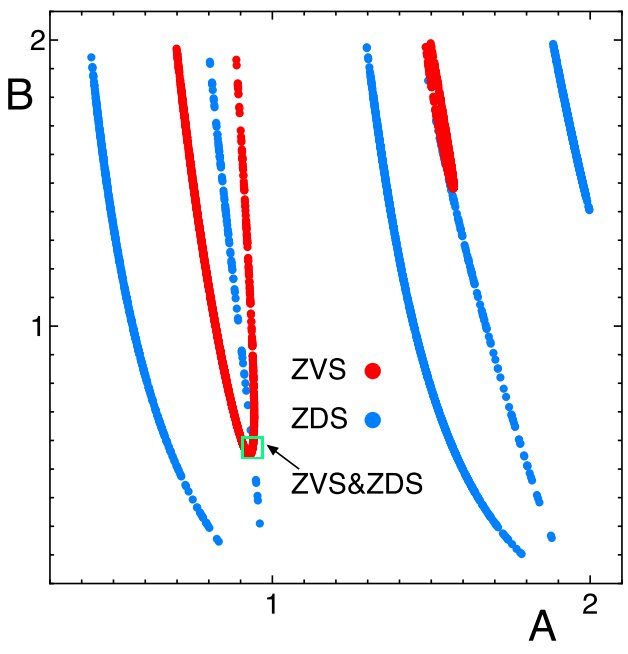

(a)

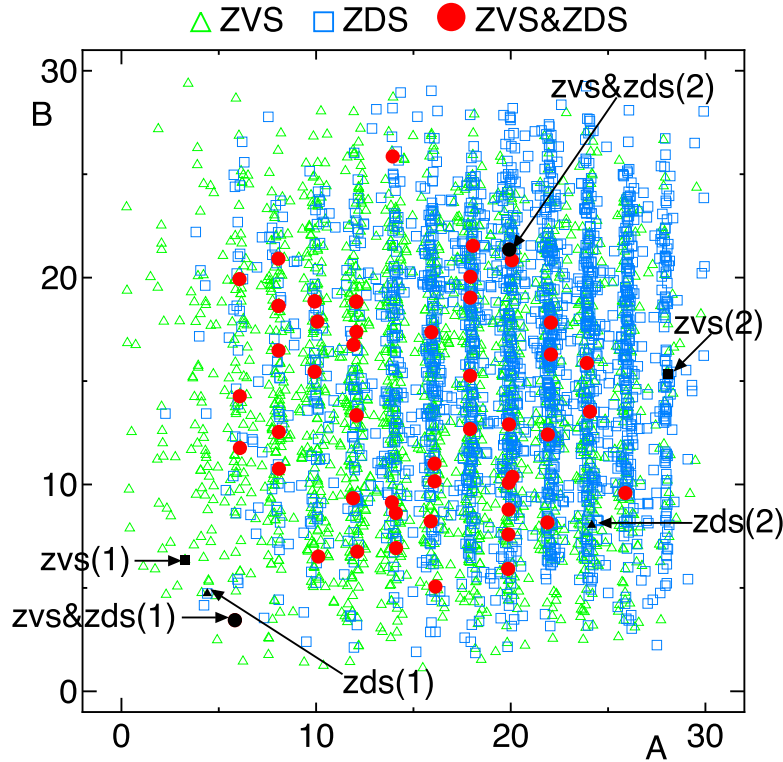

(b)

Fig. 3. Class-E ZVS and/or ZDS conditions found by PSO.

$c_{1}$, and $c_{2}$ are typical as standard PSO, and $N_{0}$ was set to 100,000 in order to find the global minimum points surely. The particles' initial positions were randomly assigned in the region. Figure 3(a) shows the global minimum points found by PSO, in which 2000 trials were run using $f_{1}(A, B)$ and $f_{2}(A, B)$, respectively, and 100 trials were run using $f_{3}(A, B)$. When an objective function value is less than $10^{-10}$, which is $\Theta_{1}$ of Table I, PSO was terminated. We found 1991 and 1965 global minimum points that satisfy the ZVS and ZDS conditions, respectively. From Fig. 3(a), the ZVS or ZDS condition appears to be satisfied not only at isolated points but on curves in the parameter space. For 32 trials, PSO found the same point that satisfies both the ZVS and ZDS conditions; thus, this point is considered to be an intersection point of two curves that respectively satisfy the ZVS and ZDS conditions. 


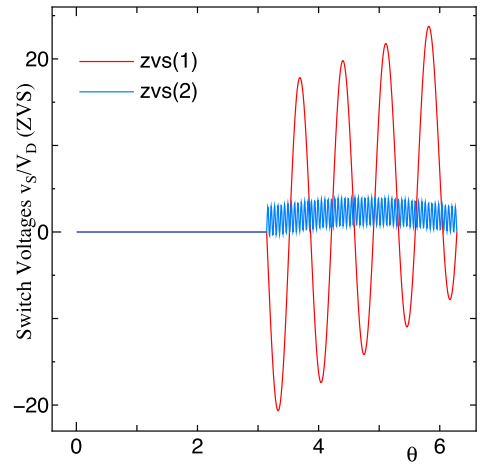

(a)

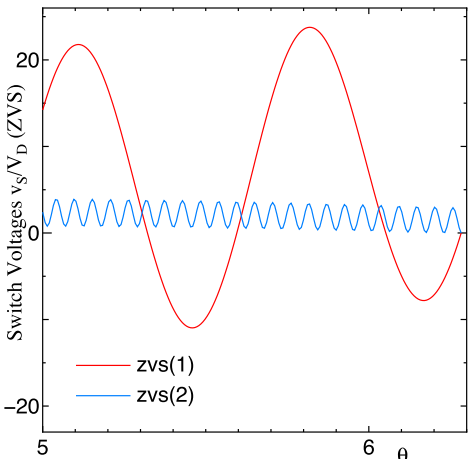

(b)

Fig. 4. Steady-state waveforms obtained by analyzing the circuits that satisfy the ZVS condition, (a) switch voltages and (b) the enlarged responses around $\theta=2 \pi$ of $(\mathrm{a})$.

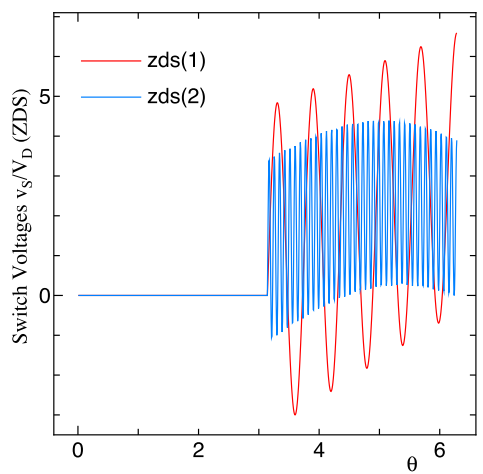

(a)

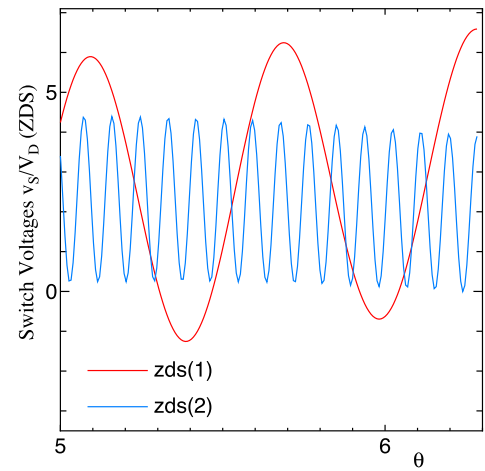

(b)

Fig. 5. Steady-state waveforms obtained by analyzing the circuits that satisfy the ZDS condition, (a) switch voltages and (b) the enlarged responses around $\theta=2 \pi$ of $(\mathrm{a})$.

Extending the searching area as $A, B \in[0.01,30.0]$, PSO was again applied, in which 2000 trials were run with each (9) and (10) and 100 trials were with (11). Figure 3(b) shows the global minimum points found by PSO, in which the ZVS and/or ZDS conditions are fulfilled at many points. Selecting the two points zvs(1) and zvs(2), we calculated the corresponding steady-state responses of the class-E amplifier. Figures $4(\mathrm{a})$ and $4(\mathrm{~b})$ show the steady-state responses of the switch voltage $\frac{v_{S}}{V_{D}}$. As shown in Fig. 4(b), the responses are zero at $\theta=2 \pi$; thus, we can confirm that the ZVS condition of (7) is satisfied on the steady-state responses. Figures $5(\mathrm{a})$ and $5(\mathrm{~b})$ show the steady-state responses of the switch voltage $\frac{v_{S}}{V_{D}}$ which satisfy the ZDS condition. The time derivatives of the responses are zero at $\theta=2 \pi$; thus, the condition (8) is satisfied on the steady-state responses. Selecting the two points zvs\&zds(1) and zvs\&zds(2) of Fig. 3(b) at which both the ZVS and ZDS conditions are satisfied, the steady-state responses of the switch voltage were calculated. As shown in Fig. 6(b), the responses and their time derivatives are both zero at $\theta=2 \pi$; thus, both the ZVS and ZDS conditions are satisfied on the steady-state responses. However, the fundamental frequency is not only $1[\mathrm{~Hz}]$ but a positive integer $[\mathrm{Hz}]$; therefore, these circuits are not class-E amplifiers and are referred to as class-E frequency multipliers. From this fact, the optimization problem with the objective function $f_{3}(A, B)$ has many global minimum points if the parameter space is not limited. Therefore, we need another criterion to obtain the class-E amplifier.

To prevent from being trapped to the frequency multiplier solutions, consider the following objective function:

$$
f_{4}(A, B)=\frac{1}{\left|V_{1}\right|} \sum_{k=0, k \neq 1}^{8}\left|V_{k}\right|,
$$

where $V_{k}$ is the $k$-th frequency component of the output voltage waveform $v_{o}$. The frequency com- 


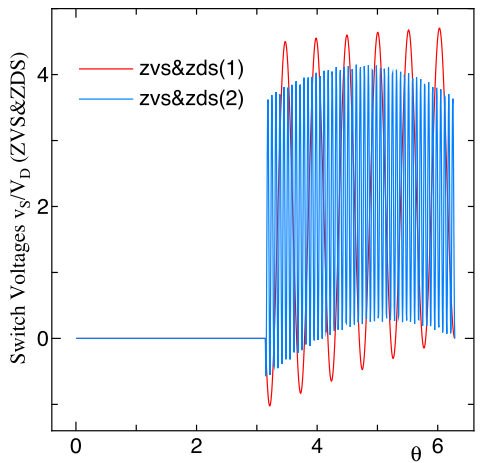

(a)

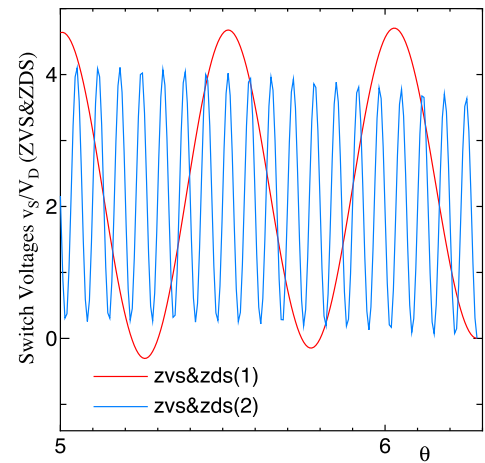

(b)

Fig. 6. Steady-state responses obtained by analyzing the circuits which satisfy both the ZVS and ZDS conditions, (a) steady-state waveforms of switch voltages, (b) the enlarged responses around $\theta=2 \pi$ of (a).

ponents are calculated by fast Fourier transform, where 16 sampled points in the time-domain are taken into account. If the DC component is eliminated, (12) is called total harmonic distortion. Note that the fundamental frequency of the output waveform is approximately $1[\mathrm{~Hz}]$ when the value of $f_{4}(A, B)$ is smaller than 1.0.

From the discussion so far, we need to find a point that minimizes $f_{1}(A, B), f_{2}(A, B)$ and $f_{4}(A, B)$ to determine the passive element values of the class-E amplifier. Further, the condition $A, B>0$ is required, because the frequencies $f_{0}$ and $f$ and the capacitances $C_{0}$ and $C_{S}$ are positive. A point which minimizes the three functions simultaneously does not exist. However, as the solution curves of $f_{1}(A, B)$ and $f_{2}(A, B)$ can exist, the points which minimize $f_{1}(A, B)$ and $f_{2}(A, B)$ simultaneously can be obtained by using $f_{3}(A, B)$ which is no-weighted sum of $f_{1}(A, B)$ and $f_{2}(A, B)$. As the output waveform contains any distortion, $f_{4}(A, B)$ does not become very small. Thus, the function $f_{4}(A, B)$ should not be added to $f_{3}(A, B)$.

Thus, the passive element values determination of the class-E amplifier is defined as a multi-objective optimization problem:

$$
\begin{array}{ll}
\text { minimize } & f_{3}(A, B), f_{4}(A, B), \\
\text { subject to } & A, B>0 .
\end{array}
$$

If there exists a tradeoff between $f_{3}(A, B)$ and $f_{4}(A, B)$, finding the Pareto solutions [19] would be a good strategy. However, even if $f_{3}(A, B)$ is small, i.e., a class-E multiplier is found, $f_{4}(A, B)$ is not necessarily large. This is because the class-E frequency multiplier may include a class-E amplifier. Therefore, as tradeoffs do not exist in this problem, we should not think to find the Pareto solutions. Additionally, the passive element values determination gives priority to the minimization with $f_{3}(A, B)$ in which the objective function value must be approximately zero. Therefore, after finding many global minimum points associated with $f_{3}(A, B)$, the point that has the smallest value of $f_{4}(A, B)$ would be selected. However, this is not efficient, because there are infinite global minimum points associated with $f_{3}(A, B)$.

Thus, we take the following strategy to determine the passive element values. First, the optimization associated with $f_{4}(A, B)$ is run. Next, the obtained minimum point is used as the initial solution to the optimization associated with $f_{3}(A, B)$, in which a computationally efficient method to find the global minimum point to a design specification is used. As we must calculate many global minimum points changing the design specification, all necessary points are calculated by applying a curve tracing algorithm.

\section{Optimization methods}

\subsection{Overview of automated design procedure}

The automated design procedure proposed in this paper is to find the design parameters $\boldsymbol{X}=[A, B]^{T}$ that satisfies the class-E ZVS and ZDS conditions of (7) and (8). Both the conditions are written by 


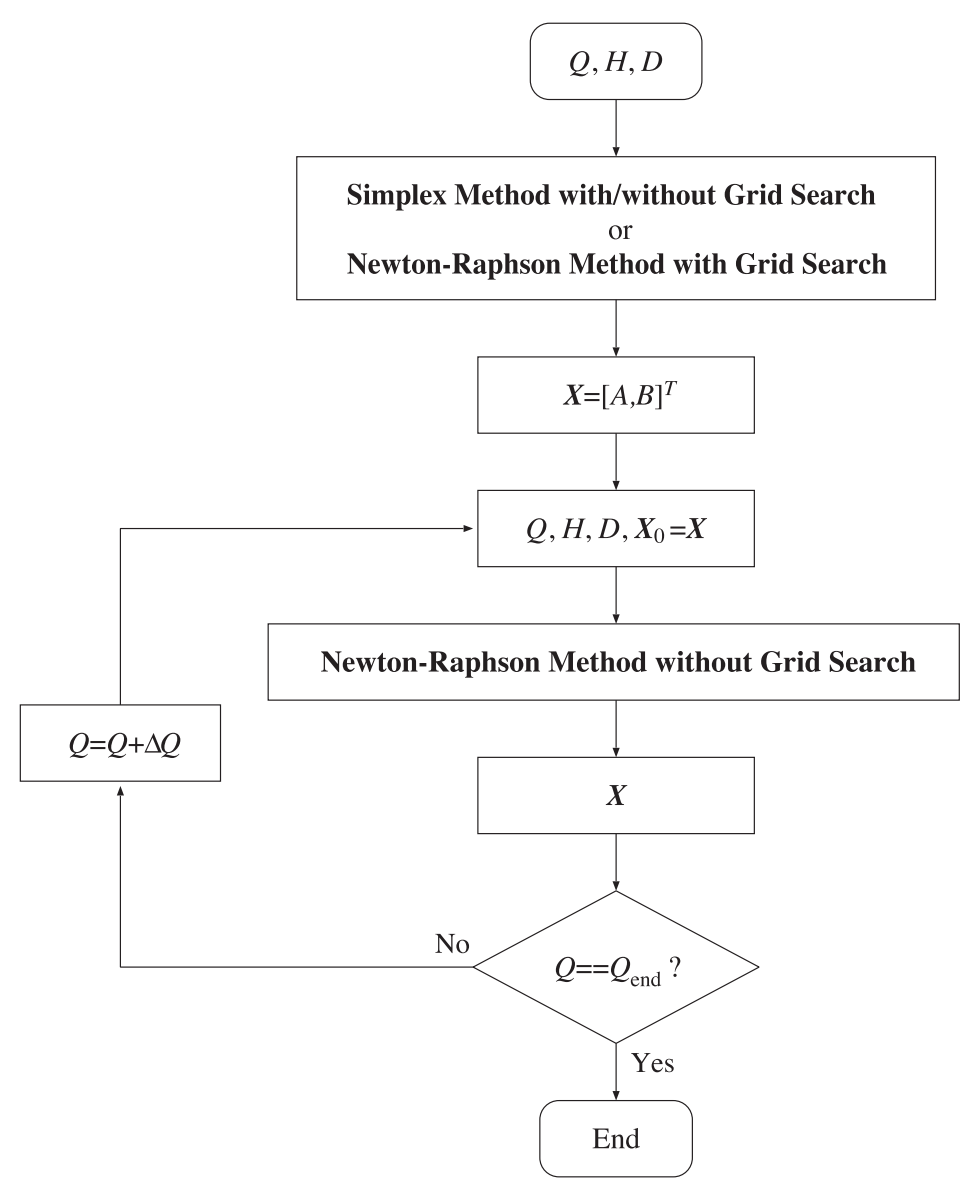

Fig. 7. Flow chart of the automated design procedure.

$$
\boldsymbol{g}(\boldsymbol{X}, Q, H, D)=\mathbf{0},
$$

where $\boldsymbol{g} \equiv\left[g_{1}, g_{2}\right]^{T}=\left[x_{2}\left(0_{-}\right), x_{1}\left(0_{-}\right)-x_{3}\left(0_{-}\right)\right]^{T}$ is associated with (6) and the solution is put as $\boldsymbol{X}=[A, B]^{T}$. Here, $(Q, H, D)$ is a design specification stated in Sect. 2.2. The solution curve of $\boldsymbol{X}=[A, B]^{T}$ that satisfies (14) is referred to as the design curve that depends on $(Q, H, D)$. The design curve depending on the loaded quality factor $Q$ only is very useful for the designers when $(H, D)$ is fixed. Giving a specification $(Q, H, D)$ and solving the optimization problem by a method, we can find a point $\boldsymbol{X}_{0}$ on the design curve. Then, changing the loaded quality factor gradually and tracing the design curve from a point $\boldsymbol{X}_{0}$, we can know the whole characteristics that satisfy both the class-E ZVS and ZDS conditions.

Figure 7 shows an overview of the automated design procedure. The point $\boldsymbol{X}_{0}$ is found by the simplex method with/without grid search or the Newton-Raphson method with grid search. The curve tracing can be done by using the Newton-Raphson method without grid search. In this section, we will explain each method step by step.

\subsection{Grid searches}

Generally, a small constrained optimization problem is solved by a grid search and a minimization algorithm [21]. Thus, applying a grid search to the objective function $f_{4}(A, B)$, the point whose objective function $f_{4}(A, B)$ is minimum over all the grid points is found. Using this point as an initial solution, a minimization algorithm is applied to find the global minimum point for the objective function $f_{3}(A, B)$.

The grid search is referred to as Grid Search 1, which is a simple two-dimensional search. The computational complexity is $O\left(N_{1}^{2}\right)$, in which $N_{1}$ is the number of divisions for each coordinate. Thus, when $N_{1}$ is large, the computational cost cannot be ignored. As the objective function $f_{4}(A, B)$ is a multimodal function, for example, $N_{1}=1000$ is used, which means that the grid search becomes 
costly. Thus, the two coordinates are searched sequentially, which is referred to as Grid Search 2, and the computational complexity is $O\left(N_{1}\right)$.

\subsection{Simplex method}

As derivative is not analytically available for the objective function $f_{3}(A, B)$, we use the simplex method [20]. In this method, a simplex is composed of $m+1$ nodes, in which $m$ represents the number of design variables. Thus, the simplex is a triangle for $m=2$. The procedure continues until the simplex projection onto the function surface rolls into a minimum point at which the $m+1$ nodes are sufficiently close together. To realize convergence, three operations, reflection, expansion, and contraction are used, evaluating the objective function values. In our implementation, we use the MATLAB function $f$ minsearch(), which is the simplex method [20]. Finding a grid point by using Grid Search 1 or Grid Search 2 and providing it as an initial solution, the simplex method is applied, which corresponds to Simplex Method with Grid Search of the flowchart of Fig. 7. Then, these algorithms are referred to as SMPLX-G1 and SMPLX-G2 which use Grid Search $\mathbf{1}$ and Grid Search 2, respectively. The MATLAB function fminsearch() has two stopping criteria $\Theta_{1}$ and $\Theta_{2}$ that are termination tolerances on the function value of (11) and the solution variation, respectively. In our implementation, we take as $\Theta_{1}=\Theta_{2}=10^{-10}$ as listed in Table I. The objective function value of (11) being less than $\Theta_{1}$ implies that the simplex method could find the global minimum point. However, satisfying the solution variation within $\Theta_{2}$ implies that the simplex method is trapped into a local minimum point without finding the global minimum point. Thus, the simplex method must be stopped, fulfilled with being less than $\Theta_{2}$.

Furthermore, we found the initial solution $\left(A^{*}, B^{*}\right)$, which is suitable for the simplex method with the objective function $f_{3}(A, B)$, as follows:

$$
\left(A^{*}, B^{*}\right)=\epsilon_{1}(1,1),
$$

where $\epsilon_{1}$ is a small positive number. This method corresponds to Simplex Method without Grid Search of the flowchart of Fig. 7 and is referred to as SMPLX. As this procedure does not require a grid search, it is very efficient. The parameter $\epsilon_{1}$ must be empirically determined.

\subsection{Newton-Raphson method}

The motivation of this paper is on the difficulty to find an initial solution for the Newton-Raphson method. In the previous subsection, the point found by a grid search is used as an initial solution for the simplex method. Fortunately, the grid point can also be used as an initial solution for the Newton-Raphson method.

Equation (14) is compactly written by

$$
g(X)=0 .
$$

After applying a grid search, we solve (16) by the Newton-Raphson method, in which the point obtained from a grid search is used as an initial solution for the Newton-Raphson method as $\boldsymbol{X}_{0}=$ $\left[A^{*}, B^{*}\right]^{T}$. To ensure the convergence of the Newton-Raphson method, the linear search with the direction

$$
\boldsymbol{d}=-\left\{\boldsymbol{g}^{\prime}\left(\boldsymbol{X}_{n}\right)\right\}^{-1} \boldsymbol{g}\left(\boldsymbol{X}_{n}\right)
$$

is used, where $\boldsymbol{g}^{\prime}\left(\boldsymbol{X}_{n}\right)$ is the Jacobian matrix. We find the smallest integer $m \geq 0$ such that

$$
\left\|\boldsymbol{g}\left(\boldsymbol{X}_{n}+2^{-m} \boldsymbol{d}\right)\right\|<\left\|\boldsymbol{g}\left(\boldsymbol{X}_{n}\right)\right\| .
$$

Then, the solution is written by

$$
\boldsymbol{X}_{n+1}=\boldsymbol{X}_{n}+2^{-m} \boldsymbol{d} .
$$

The linear search (18) is truncated until $N_{3}$ iterations. If $m$ is not found, put $m=0$. 
The elements of the Jacobian matrix are obtained by giving a small perturbation $\epsilon_{2}$ to the design parameters and calculating (6). For example, $\partial g_{1} / \partial X_{1}$, which is the $(1,1)$ element, is calculated by

$$
\frac{\partial g_{1}}{\partial X_{1}}=\frac{g_{1}\left(A+\epsilon_{2}, B\right)-g_{1}(A, B)}{\epsilon_{2}} .
$$

This method corresponds to Newton-Raphson Method with Grid Search of the flowchart of Fig. 7. When Grid Search 2 is used, the Newton-Raphson method is referred to as NEW-G2. Note that the Newton-Raphson method itself is not necessarily useful for finding a set of design parameters, but is effectively incorporated with the curve tracing algorithm provided in the next subsection in order to calculate many sets of design parameters changing the design specification.

\subsection{Design curve tracing}

After applying the Simplex method or the Newton-Raphson method, the curve tracing is started with the initial solution $\boldsymbol{X}=[A, B]^{T}$, which is given by one of these methods, as shown in Fig. 7. Then, the solution $\boldsymbol{X}=[A, B]^{T}$ is used as the initial solution of the Newton-Raphson method (NewtonRaphson Method without Grid Search of Fig. 7) as $\boldsymbol{X}_{0}=\boldsymbol{X}$. After the Newton-Raphson method converges, the solution is given as the initial solution for the Newton-Raphson method at the next $\mathrm{Q}$ value. Changing the loaded quality factor $\mathrm{Q}$ gradually, we can trace the design curve until $Q_{\text {end }}$. Then, all the necessary values of $(A, B)$ s are obtained; thus, the passive element values of the class-E amplifier can be determined for every needed specification.

\section{Results}

All the results throughout this paper were performed on a computer with a 3.7-GHz Intel Xeon E5-1620 CPU and 32 GB of memory, using MATLAB R2017a.

\subsection{Methods with/without grid searches}

The grid searches of Algorithms 1 and $\mathbf{2}$ for $\left[x_{\max }, x_{\min }\right]=[0.01,100]$ were used with the simplex method, in which the specification was given as $Q=30, H=1$, and $D=0.5$. Table II shows the found points obtained by the grid searches associated with the objective function (12). As the points obtained by Grid Search 1 are the same as those by Grid Search 2, the final points found by SMPLX-G1 are the same as those by SMPLX-G2. Thus, the function values of (11) and (12) for SMPLX-G1 are identical to those for SMPLX-G2. The underline indicates that an appropriate minimum point as the class-E amplifier is not obtained. Note that the parameters $\Theta_{2}, N_{0}$, and $N_{2}$ are set to terminate the simplex method and these parameters do not improve the performance of the simplex method. Only when $N_{1}=1000$, the simplex method provided a global minimum point suitable for the class-E amplifier. When $N_{1}=10$, the simplex method did not converge. For the other

\footnotetext{
Algorithm 1 Grid Search 1

1. Divide $\left[x_{\min }, x_{\max }\right]$ uniformly for each ordinate, in which $N_{1}$ is the number of divisions.

2. Find the point whose objective function value of (12) is the smallest within all the grid points $\left(A_{i}, B_{j}\right), i, j=0,1, \ldots, N_{1}$ and put it as $\left(A^{*}, B^{*}\right)$.
}

\section{Algorithm 2 Grid Search 2}

1. Divide $\left[x_{\min }, x_{\max }\right]$ uniformly for each ordinate, in which $N_{1}$ is the number of divisions.

2. Find the point whose objective function value of (12) is the smallest within the grid points $\left(A_{i}, x_{\min }\right), i=0,1, \ldots, N_{1}$ and put it as $\left(A^{*}, x_{\min }\right)$.

3. Find the point whose objective function value of (12) is the smallest within the grid points $\left(A^{*}, B_{j}\right), j=0,1, \ldots, N_{1}$ and put it as $\left(A^{*}, B^{*}\right)$. 
Table II. Function values of (11) and (12) obtained from the simplex method with grid searches and the computational times of these methods.

(a)

\begin{tabular}{|c||c|c|c|c|c|}
\hline \multicolumn{1}{|c||}{} & \multicolumn{2}{c|}{ Grid Search 1 } & \multicolumn{3}{c|}{ SMPLX-G1 } \\
\hline$N_{1}$ & minimum point & time [sec.] & $(11)$ & $(12)$ & time [sec.] \\
\hline \hline 1000 & $(1.0099,0.11000)$ & 2436.64 & $2.8 \times 10^{-11}$ & 0.021 & 0.065 \\
\hline 500 & $(1.0099,0.01)$ & 583.62 & $5.1 \times 10^{-2}$ & $\underline{2.650}$ & 0.071 \\
\hline 100 & $(1.0099,0.01)$ & 22.76 & $5.1 \times 10^{-2}$ & $\underline{2.650}$ & 0.073 \\
\hline 10 & $(0.01,100.0)$ & 0.27 & $1.0 \times 10^{-1}$ & $\underline{0.259}$ & 3.061 \\
\hline
\end{tabular}

(b)

\begin{tabular}{|c||c|c|c|c|c|}
\hline \multicolumn{1}{|c||}{} & \multicolumn{2}{c|}{ Grid Search 2 } & \multicolumn{3}{c|}{ SMPLX-G2 } \\
\hline$N_{1}$ & minimum point & time [sec.] & $(11)$ & $(12)$ & time [sec.] \\
\hline \hline 1000 & $(1.0099,0.11000)$ & 4.359 & $2.8 \times 10^{-11}$ & 0.021 & 0.074 \\
\hline 500 & $(1.0099,0.01)$ & 2.175 & $5.1 \times 10^{-2}$ & $\underline{2.650}$ & 0.088 \\
\hline 100 & $(1.0099,0.01)$ & 0.445 & $5.1 \times 10^{-2}$ & $\underline{2.650}$ & 0.079 \\
\hline 10 & $(0.01,100.0)$ & 0.054 & $1.0 \times 10^{-1}$ & $\underline{0.259}$ & 3.114 \\
\hline
\end{tabular}

Table III. Values of (12) obtained from SMPLX, SMPLX-G2, and NEW-G2, (a) $H=1$, (b) $H=0.001$, (c) $H=10^{-10}$.

\begin{tabular}{|c|c|c||c|c|}
\hline & $\begin{array}{c}\text { SMPLX-G2 } \\
\text { /NEW-G2 }\end{array}$ & SMPLX & $\begin{array}{c}\text { SMPLX-G2 } \\
\text { /SMPLX }\end{array}$ & NEW-G2 \\
\hline \hline$Q$ & $N_{1}$ & $\epsilon_{1}$ & \multicolumn{2}{|c|}{$(12)$} \\
\hline 30 & 1000 & 0.01 & 0.021 & 0.021 \\
\hline 20 & 1000 & 0.01 & 0.032 & 0.032 \\
\hline 10 & 1000 & 0.01 & 0.061 & $\underline{0.76}$ \\
\hline 5 & 1000 & 0.01 & 0.114 & 0.114 \\
\hline 3 & 1000 & 0.05 & 0.176 & 0.176 \\
\hline 2 & 1000 & 0.05 & 0.244 & 0.244 \\
\hline
\end{tabular}

(b)

\begin{tabular}{|c|c|c||c|c|}
\hline & $\begin{array}{c}\text { SMPLX-G2 } \\
\text { /NEW-G2 }\end{array}$ & SMPLX & $\begin{array}{c}\text { SMPLX-G2 } \\
\text { /SMPLX }\end{array}$ & NEW-G2 \\
\hline \hline$Q$ & $N_{1}$ & $\epsilon_{1}$ & \multicolumn{2}{|c|}{$(12)$} \\
\hline 30 & 1000 & 0.01 & 0.022 & $\underline{0.801}$ \\
\hline 20 & 1000 & 0.01 & 0.033 & 0.033 \\
\hline 10 & 1000 & 0.01 & 0.066 & 0.066 \\
\hline 5 & 1000 & 0.01 & 0.133 & 0.133 \\
\hline 3 & 1000 & 0.01 & 0.225 & 0.225 \\
\hline 2 & 500 & 0.05 & 0.349 & 0.349 \\
\hline
\end{tabular}

(c)

\begin{tabular}{|c|c|c||c|c|}
\hline & $\begin{array}{c}\text { SMPLX-G2 } \\
\text { /NEW-G2 }\end{array}$ & SMPLX & $\begin{array}{c}\text { SMPLX-G2 } \\
\text { /SMPLX }\end{array}$ & NEW-G2 \\
\hline \hline$Q$ & $N_{1}$ & $\epsilon_{1}$ & \multicolumn{2}{|c|}{$(12)$} \\
\hline 30 & 1000 & 0.9 & 0.022 & $\underline{2.758}$ \\
\hline 20 & 1000 & 0.1 & 0.033 & 0.033 \\
\hline 10 & 1000 & 0.1 & 0.066 & 0.066 \\
\hline 5 & 1000 & 0.1 & 0.133 & 0.133 \\
\hline 3 & 1000 & 0.1 & 0.225 & 0.225 \\
\hline 2 & 500 & 0.1 & 0.350 & 0.350 \\
\hline
\end{tabular}




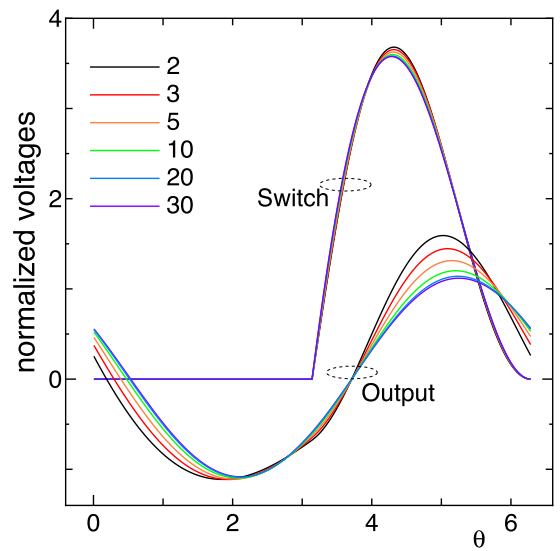

(a)

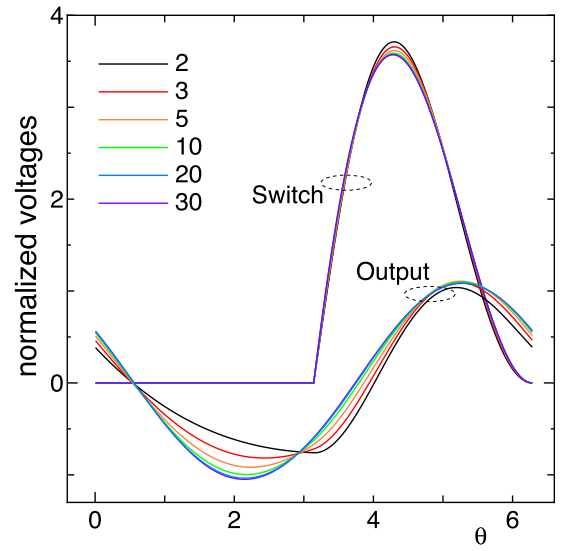

(b)

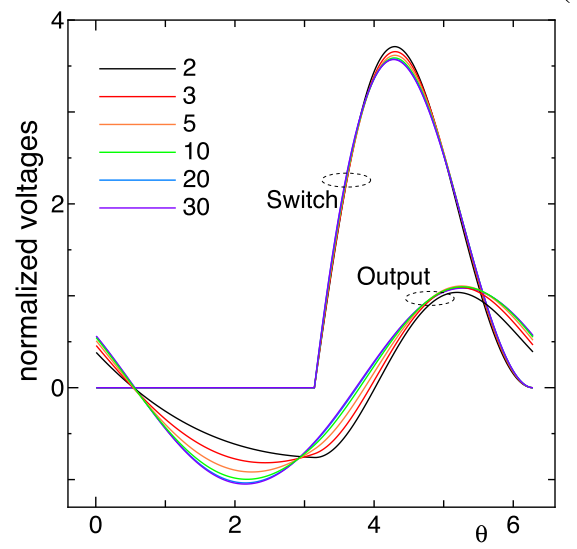

(c)

Fig. 8. Steady-state switch and output waveforms, $v_{s} / V_{D}$ and $R i_{o} / V_{D}$, respectively, (a) $H=1$, (b) $H=0.001$, (c) $H=10^{-10}$.

cases, the simplex method converged within 200 function evaluations; thus, the computational times are very short except for $N_{1}=10$. When $N_{1}=100$ and $N_{1}=500$, the simplex method terminated with filling the condition $\Theta_{2}$, which implies that the simplex method was trapped to a local minimum. As Grid Search 2 is much faster than Grid Search 1, SMPLX-G2 is preferable to SMPLX-G1.

Table III shows the values of (12) obtained by SMPLX, SMPLX-G2 and NEW-G2, in which $\epsilon_{1}$ is set for SMPLX and $N_{1}$ is for SMPLX-G2 and NEW-G2. For SMPLX-G2 and NEW-G2, $\left[x_{\max }, x_{\min }\right]=$ $[0.01,100]$ were also assumed. From Table III, SMPLX provides the same values of (12) as SMPLXG2. However, NEW-G2, which is the Newton-Raphson method provided in Sect. 3.4, did not converge for some cases where $N_{4}=2000$ was set, which implies that the simplex method is superior to the Newton-Raphson method.

For SMPLX-G2 and NEW-G2, we must set $x_{\min }, x_{\max }$, and $N_{1}$, and the grid search is required. For SMPLX, we have only to set $\epsilon_{1}$ and any grid search is not required. Thus, SMPLX is the most effective for solving this problem and we use SMPLX for the design examples in the following subsections. Figure 8 shows the normalized switch and output voltage waveforms by $V_{D}$, which are obtained by using the parameters provided by SMPLX. From all the switch voltage waveforms at $\theta=2 \pi$, the ZVS and ZDS conditions are satisfied for all the cases; thus, the passive elements of class-E amplifiers were adequately determined.

\subsection{Design Example 1}

We used the example provided in [18] to confirm the accuracy of the curve tracing algorithm in Sect. 4, where $H=0$ is assumed. However, as we cannot assign infinite $L_{C}$ to solve the circuit equations numerically, $H=1.0 \times 10^{-10}$ was used.

We calculated some factors depending on the loaded quality factor, in which $Q$ was divided in three regions: $[1.7879,3],[3,20]$, and $\left[20,5 \times 10^{5}\right]$. For $[1.7879,3]$, the solution at $Q=3$ was first calculated 


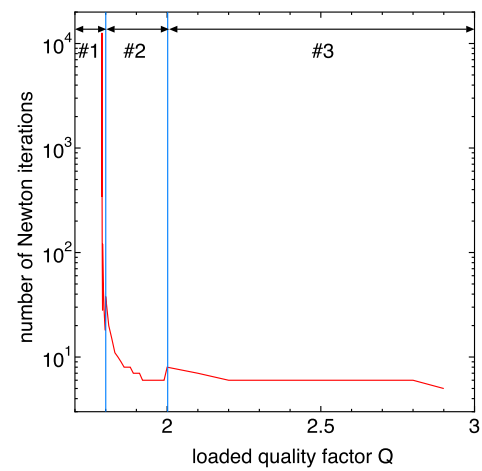

(a)

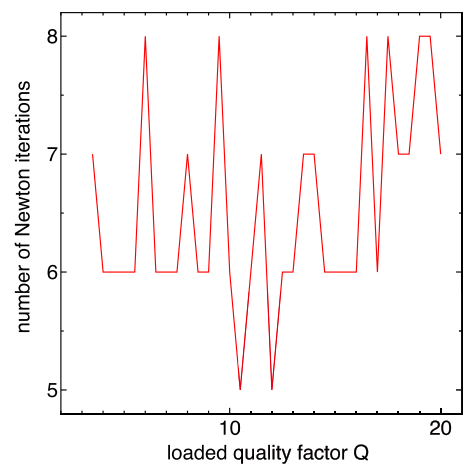

(b)

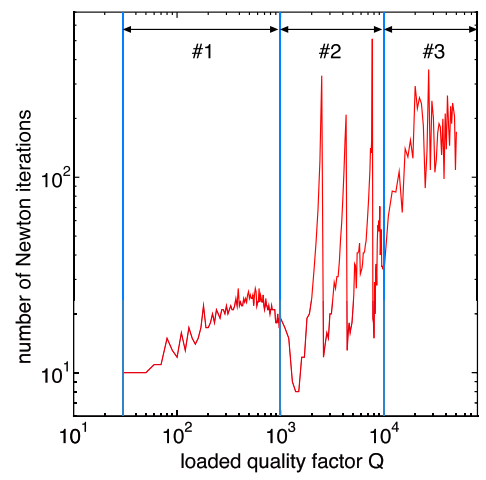

(c)

Fig. 9. Numbers of iterations of Newton-Raphson method needed to find the solutions, (a) $Q \in[1.7879,3)$, (b) $Q \in[3,20)$, (c) $Q \in\left[20,5 \times 10^{5}\right]$.

Table IV. Some factors depending on loaded quality factor $Q$.

\begin{tabular}{|c||c|c|c|c|c|c|}
\hline \multicolumn{1}{|c||}{$Q$} & \multicolumn{2}{c|}{$P R / V_{D}^{2}$} & \multicolumn{2}{c|}{$C_{S} \omega R$} & \multicolumn{2}{c|}{$C_{0} \omega R$} \\
\hline & Reference [18] & Proposed & Reference [18] & Proposed & Reference [18] & Proposed \\
\hline \hline$\infty$ & 0.57680 & 0.57680 & 0.18360 & 0.18360 & 0 & 0.00002 \\
\hline 20 & 0.56402 & 0.56397 & 0.19111 & 0.19111 & 0.05313 & 0.05313 \\
\hline 10 & 0.54974 & 0.54963 & 0.19790 & 0.19790 & 0.11375 & 0.11375 \\
\hline 5 & 0.51659 & 0.51662 & 0.20907 & 0.20907 & 0.26924 & 0.26924 \\
\hline 3 & 0.46453 & 0.46452 & 0.21834 & 0.21834 & 0.63467 & 0.63467 \\
\hline 2.5 & 0.43550 & 0.43550 & 0.22036 & 0.22036 & 1.01219 & 1.01219 \\
\hline 2 & 0.38888 & 0.38888 & 0.21994 & 0.21994 & 3.05212 & 3.05212 \\
\hline 1.7879 & 0.35969 & 0.35980 & 0.21770 & 0.21770 & $\infty$ & $3,157.1$ \\
\hline
\end{tabular}

In the proposed method, $Q=5 \times 10^{4}$ was used instead of $\infty$ to calculate each factor.

by the simplex method, and the characteristics curve was traced by the Newton-Raphson method in Sect. 3.5. For the simplex method (SMPLX), the initial solution was given as $(A, B)=\left(10^{-2}, 10^{-2}\right)$. In the Newton-Raphson method, $Q$ was decremented with $10^{-1}$ until $2,10^{-2}$ until $1.8,10^{-3}$ until $1.79,10^{-4}$ until 1.7881 , and $2.0 \times 10^{-5}$ until 1.7879. As a perturbation for calculating the elements of Jacobian matrix in $(20), \epsilon_{2}=10^{-3}$ was used until $1.79, \epsilon_{2}=10^{-5}$ until 1.7881, and $\epsilon_{2}=10^{-10}$ until 1.7879 .

For $[3,20]$, the solution at $Q=3$ was first calculated by the simplex method. Incrementing $Q$ with 0.5 , we calculated the design curve. For $\left[20,5 \times 10^{5}\right]$, the solution at $Q=20$ was first calculated by the simplex method, and $Q$ was incremented with $10^{2}$ until $10^{3}, 10^{3}$ until $10^{4}$, and $5 \times 10^{3}$ until $5 \times 10^{4}$. The perturbation $\epsilon_{2}=10^{-3}$ was taken for the Newton-Raphson method.

The Newton-Raphson method was terminated when $f_{3}(A, B)<\Theta_{1}=10^{-10}$ or $N_{4}=20000$. The other parameters are listed in Table I. Note that the parameters for the curve tracing algorithm are set to obtain the design parameters at strange $Q$ 's. Figures 9 (a) and 9 (c) show the numbers of iterations needed to find the solution at each step, in which the intervals \#1, \#2, and \#3 respectively imply that an uniform incremental of $Q$ was used within the interval. When $Q$ is small or large, a large number of iterations are required for the Newton-Raphson method to converge, because the design parameters $(A, B)$ abruptly change when $Q$ is small in Fig. 9(a) and the incremental $Q$ is taken largely when $Q$ is large in Fig. 9(c).

To compare with the results [18], the output (average) power $P$ and voltage $V_{o}$ are respectively defined as

$$
P=\frac{V_{o}^{2}}{R}, \quad V_{o}=\sqrt{\frac{1}{2 \pi} \int_{0}^{2 \pi} v_{o}^{2}(t) d t},
$$

where the integral related to $V_{0}$ was calculated by a trapezoidal rule.

Table IV shows values of $P R / V_{D}^{2}, C_{S} \omega R$, and $C_{0} \omega R$ on $Q$, where $R=5[\Omega]$ and $V_{D}=5[\mathrm{~V}]$ were 


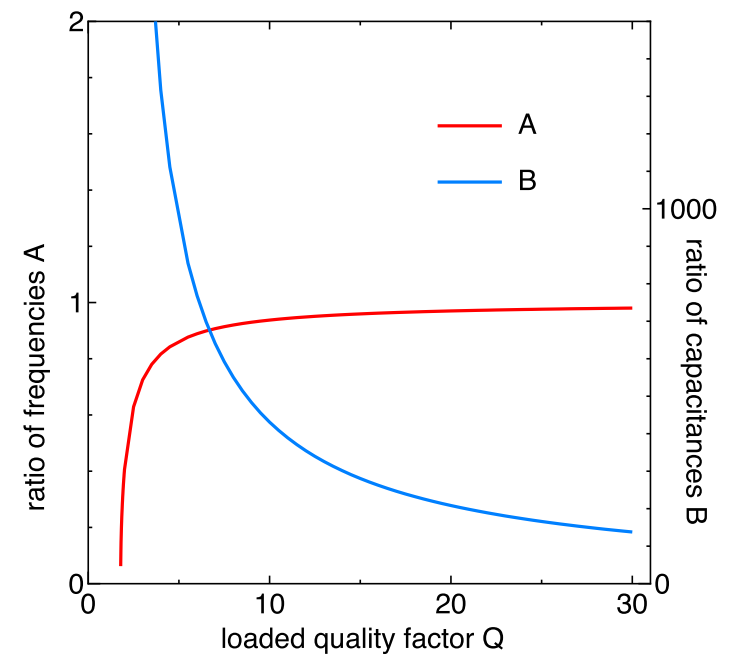

(a)

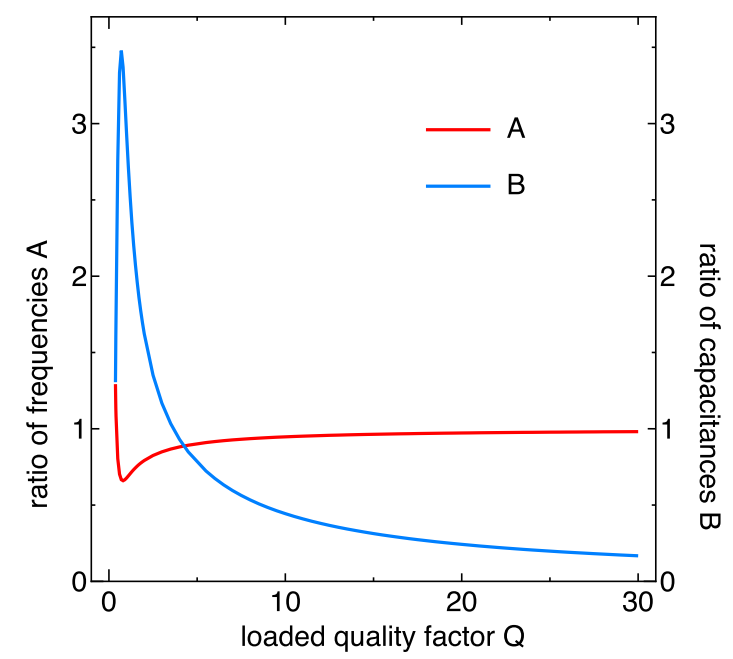

(b)

Fig. 10. Design characteristics of Example 2, (a) $H=0.001$ and (b) $H=1$.

assumed, and the last two values were calculated as

$$
C_{S} \omega R=\frac{1}{A^{2} B Q}, \quad C_{0} \omega R=\frac{1}{A^{2} Q} .
$$

The results obtained from the proposed method are almost identical to [18].

\subsection{Design Example 2}

As the second design example, we consider the case of $H \neq 0$, whereas $H \approx 0$ is assumed in the previous design example. All the parameters $(A, B)$ depending on $Q$ were calculated by the curve tracing algorithm, in which the initial solution to trace the curve was calculated by the simplex method.

Figure 10 shows the design characteristics. To obtain them, the solution at $Q=5$ was first calculated by the simplex method with the initial solution $(A, B)=\left(10^{-2}, 10^{-2}\right)$ and the design curve was traced to both directions using the Newton-Raphson method. For $H=0.001$, incrementing $Q$ with 0.5 and oppositely decrementing with 0.5 until $Q=2$ and 0.01 until $Q=1.79$, we traced the curve. At $Q=1.78$, the Newton-Raphson method did not converge. For $H=1$, incrementing $Q$ with 0.5 and oppositely decrementing with 0.5 until $Q=2$ and 0.01 until $Q=0.37$, we traced the curve. At $Q=0.36$, the Newton-Raphson method did not converge. Therefore, we can calculate the design characteristics for the case of $H \neq 0$ except for strange $Q$ values.

\subsection{PSO and IPSO}

Finally, PSO and IPSO with the objective function $f_{3}(A, B)$ were applied to find the passive element values of the class-E amplifier. With several $x_{\min }$ and $x_{\max }$, PSO and IPSO were evaluated over 100 trials, in which the design specification was given as $Q=10, H=1$, and $D=0.5$. If the objective function value of $(11)$ is less than $\Theta_{1}$ within $N_{0}$ iterations and the global best position is $\left(p_{g 1}, p_{g 2}\right)=(0.9813986,0.1675134)$, the circuit satisfies the ZVS and ZDS conditions and the class-E amplifier is obtained. On the other hand, when the global best position is not $(0.9813986,0.1675134)$, the circuit corresponds to a class-E frequency multiplier.

Table V shows the performances of PSO and IPSO, in which "amplifier" indicates the percentage that the class-E amplifier is obtained and "multipliers" indicates the percentage that class-E frequency multipliers are obtained. PSO found the appropriate global minimum point as the class-E amplifier in only one trial and often converged into the global minimum points that correspond to the class-E frequency multipliers. IPSO could not find any global minimum point. From this fact, we can say that the passive element values of the class-E amplifier are not easily determined with the objective function (11) only. From the experiment results, we conclude that the proposed optimization procedure is more reliable than a metaheuristic method such as PSO. 
Table V. Rates [\%] that PSO and IPSO found the minimum points that correspond to the class-E amplifier or frequency multipliers.

\begin{tabular}{|c|c||c|c|c|c|}
\hline & \multicolumn{1}{|c||}{} & \multicolumn{2}{c|}{ PSO } & \multicolumn{2}{c|}{ IPSO } \\
\hline$x_{\min }$ & $x_{\max }$ & amplifier [\%] & multipliers [\%] & amplifier [\%] & multipliers [\%] \\
\hline \hline 0.01 & 100 & 0 & 0 & 0 & 0 \\
\hline 0.01 & 100 & 0 & 11 & 0 & 0 \\
\hline 0.1 & 10 & 0 & 22 & 0 & 0 \\
\hline 0.1 & 1 & 1 & 0 & 0 & 0 \\
\hline
\end{tabular}

\section{Conclusions}

We have presented an automated design procedure for class-E amplifier. First, an efficient steady-state analysis of the class-E amplifier is presented, which provides a behavioral model that is used to evaluate the class-E ZVS and ZDS conditions efficiently. Without relying on the analytical expressions of the class-E amplifier, the passive element values determination is defined as an optimization problem. Using the simplex method, a minimization algorithm is proposed, which is more reliable than the IPSO-based method [13]. To calculate the design parameters changing the design specification, the curve tracing algorithm is provided using the Newton-Raphson method. Consequently, the proposed optimization procedure neither requires many computational resources nor relies on the analytical expressions that the designers with expert knowledge of the class-E amplifier can only derive.

We can apply the proposed method to other resonant power converter and amplifier designs [16]. Moreover, the proposed method can be easily linked to a circuit simulator [17]; then, the nonlinear effects of active devices are taken into account within a circuit simulation level.

\section{Acknowledgments}

This work was partly supported by JSPS KAKENHI Grant Number 19K12004.

\section{Appendix}

In this section, we explain IPSO [13] which is applied to solve the optimization problem with the objective function $f_{3}(A, B)$. IPSO is an extension of PSO which thus is a special case of IPSO.

In PSO, there are multiple potential solutions, which are called "particles". At each step, each particle flies toward its own past best position (pbest) as well as the best position among all the particles (gbest). Thus, all the particles of (standard) PSO are always fully-connected and affect one another. IPSO particles possess independence; thus, IPSO includes isolated particles that are not connected to any particle, and the connections among particles are stochastically determined at every step. Each particle has two kinds of information, position and velocity. The position and velocity of particle $i$ are represented by $\boldsymbol{X}_{i}=\left(x_{i 1}, x_{i 2}\right)$ and $\boldsymbol{Y}_{i}=\left(y_{i 1}, y_{i 2}\right)$, respectively. Figure A-1 shows a pseudo code, in which $d=1,2$ indicates a coordinate of particle's position. Steps of IPSO are summarized as follows:

(Step1) (Initialization) Let the iteration step $n$ be 0 . Initialize the particle position $\boldsymbol{X}_{i}\left(x_{i d} \in\right.$ $\left.\left[x_{\min }, x_{\max }\right]\right)$, which is randomly selected in the parameter space, its velocity as $\boldsymbol{Y}_{i}=\mathbf{0}$, and $\boldsymbol{P}_{i}=$ $\left(p_{i 1}, p_{i 2}\right)=\boldsymbol{X}_{i}$.

(Step2) Decide a connection relationship for every coordinate. In the $d$-th coordinate, each particle $i$ is connected to the others with a probability $K ; i \in S_{C d}$, where $S_{C d}$ is a set of particles connected to the swarm in the $d$-th coordinate. In other words, the particle $i$ is isolated from the swarm with a probability $1-K$, and the particle $i$ does not interact with the others. $K \in[0.01,0.1]$, which is a constant cooperative coefficient, is effective for most problems [12].

(Step3) Evaluate the objective function $f_{3}\left(\boldsymbol{X}_{i}\right)$ for each particle $i$. Update the personal best position (pbest) as $\boldsymbol{P}_{i}=\boldsymbol{X}_{i}$, if $f_{3}\left(\boldsymbol{X}_{i}\right)<f_{3}\left(\boldsymbol{P}_{i}\right)$.

(Step4) Let $\boldsymbol{P}_{l}$ represent the best position lbest with the local best pbest among the connected particles in $S_{C}=\cup_{d} S_{C d}$. The standard PSO always uses gbest for updating the particle information. However, IPSO uses lbest instead of gbest, because the connection relationships change at every step. 


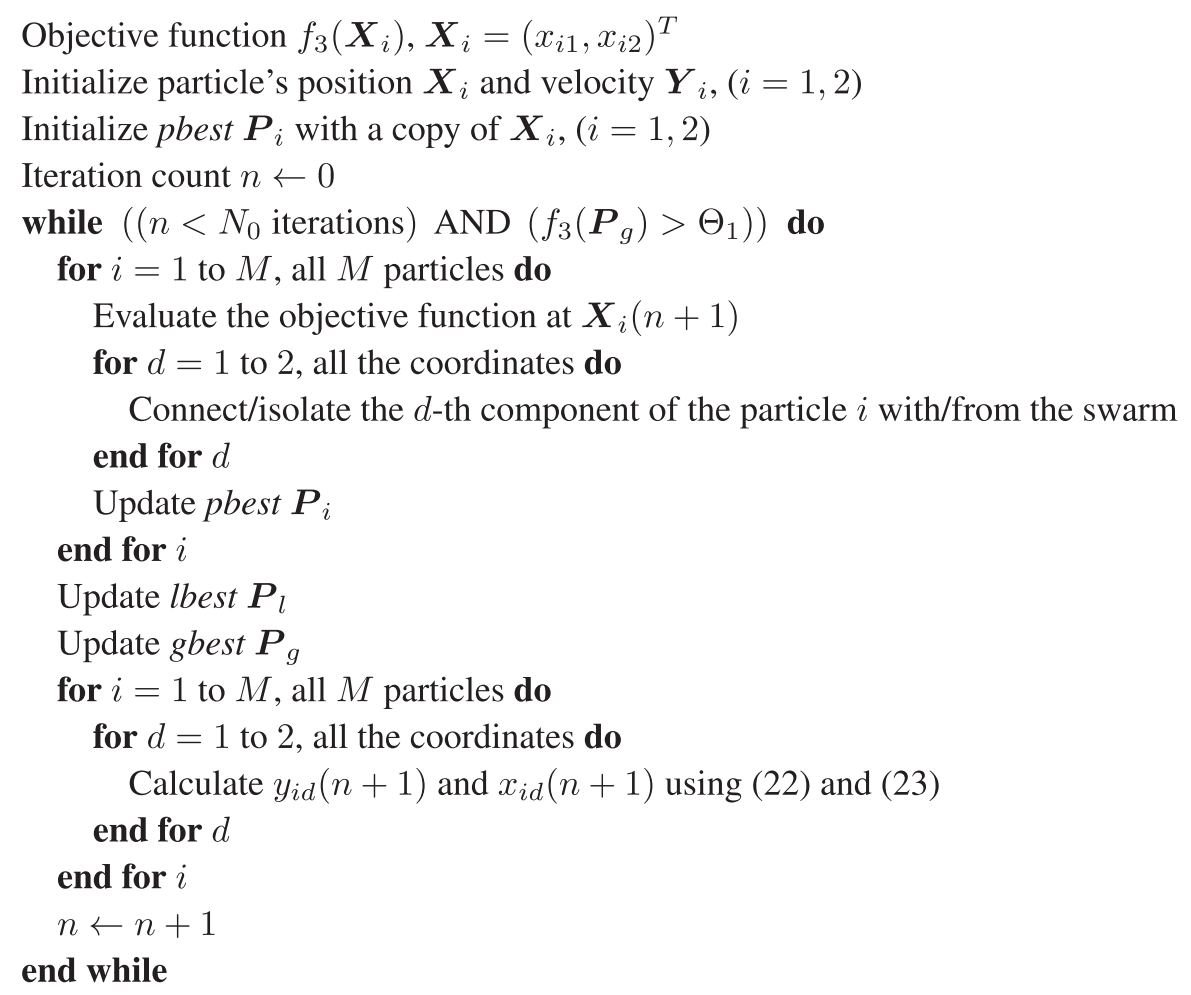

Fig. A-1. Pseudo code of IPSO.

Update lbest as $\boldsymbol{P}_{l}=\left(p_{l 1}, p_{l 2}\right)$ according to

$$
l=\arg \min _{i} f_{3}\left(\boldsymbol{P}_{i}\right), \quad i \in S_{C} .
$$

Note that the particles isolated from the swarm do not affect the new lbest, even if gbest is the minimum pbest among all the particles including the isolated particles.

(Step5) Update $\boldsymbol{Y}_{i}$ and $\boldsymbol{X}_{i}$ according to

$$
\begin{aligned}
& y_{i d}(n+1)=\left\{\begin{array}{cc}
w y_{i d}(n)+c_{1} r_{1}\left(p_{i d}-x_{i d}(n)\right) & \\
+c_{2} r_{2}\left(p_{l d}-x_{i d}(n)\right), & \in S_{C d} \\
w y_{i d}(n)+c_{1} r_{1}\left(p_{i d}-x_{i d}(n)\right), & \text { otherwise }
\end{array}\right. \\
& x_{i d}(n+1)=x_{i d}(n)+y_{i d}(n+1),
\end{aligned}
$$

where $w$ is the inertia weight, $c_{1}$ and $c_{2}$ are positive acceleration coefficients, $c_{1}=c_{2}$ in general, and $r_{1}$ and $r_{2}$ are uniform random numbers $\in[0.01,0.1]$, which are represented as $U(0,1)$. In (A-2), we stochastically determine whether each particle is affected by lbest or not, by using the cooperativeness $K$. When $K=0$, all the particles move depending only on pbest, and when $K=1$, the algorithm is entirely the same with (standard) PSO.

(Step6) Let $n=n+1$, and go back to (Step2) if any of stopping conditions ( $n==N_{0}$ OR $\left.f_{3}\left(\boldsymbol{P}_{g}\right)<\Theta_{1}\right)$ are satisfied, where $\boldsymbol{P}_{g}=\left(p_{g 1}, p_{g 2}\right)$ is gbest.

\section{References}

[1] H. Sekiya, T. Ezawa, and Y. Tanji, "Design procedure for class E switching circuits allowing implicit circuit equations," IEEE Trans. Circuits Syst. I, vol. 55, no. 11, pp. 3688-3696, December 2008.

[2] N.O. Sokal and A.D. Sokal, "Class E-A new class of high-efficiency tuned single-ended switching power amplifiers," IEEE J. Solid-State Circuits, vol. SSC-10, no. 3, pp. 168-176, June 1975. 
[3] F.H. Raab, "Idealized operation of the class E tuned power amplifier," IEEE Trans. Circuits Syst., vol. CAS-24, no. 12, pp. 725-735, December 1977.

[4] M.K. Kazimierczuk and K. Puczko, "Exact analysis of class E tuned power amplifier at any Q and switch duty cycle," IEEE Trans. Circuits Syst., vol. CAS-34, no. 2, pp. 149-159, February 1987.

[5] M.K. Kazimierczuk, "Class E tuned power amplifier with nonsinusoidal output voltage," IEEE J. Solid-State Circuits, vol. SSC-21, no. 2, pp. 687-691, February 1987.

[6] S.H. Tu and C. Toumazou, "Effect of the loaded quality factor on power efficiency for CMOS class-E RF tuned power amplifiers," IEEE Trans. Circuits Syst. I, vol. 46, no. 5, pp. 628-634, May 1999.

[7] A. Mediano, P. Molina-Gaud, and C. Bernal, "Design of class E amplifier with nonlinear and linear shunt capacitances for any duty cycle," IEEE Trans. Microw. Theory Tech., vol. 55, no. 3, pp. 484-492, April 2004.

[8] T. Suetsugu and M.K. Kazimierczuk, "Analysis and design of class E amplifier with shunt capacitance composed of nonlinear and linear capacitances," IEEE Trans. Circuits Syst. I, vol. 51, no. 7, pp. 1261-1268, July 2004.

[9] H. Sekiya, I. Sasase, and S. Mori, "Computation of design values for class E amplifiers without using waveform equations," IEEE Trans. Circuits Syst. I, vol. 49, no. 7, pp. 966-978, July 2002.

[10] R. Poli, J. Kennedy, and T. Blackwell, "Particle swarm optimization -an overview," Swarm Intell, vol. 1, pp. 33-57, 2007.

[11] Y. Tanji, H. Matsushita, and H. Sekiya, "Particle swarm optimization for design of class-E amplifier," Nonlinear Theory and Its Applications, IEICE, vol. 3, no. 4, pp. 586-595, 2012.

[12] H. Matsushita, Y. Nishio, and T. Saito, "Application of independent-minded particle swarm optimization to parameter search in switched dynamical systems," Proc. NOLTA'11, pp. 631635, September 2011.

[13] H. Matsushita and Y. Tanji, "Application of independent-minded particle swarm optimization for design of class-E amplifiers," Proc. 6th International Conference on Soft Computing and Intelligent Systems and The 13th International Symposium on Advanced Intelligent Systems, pp. 60-64, November 2012.

[14] P. Reynaert, K.L.R. Mertens, and M.S.J. Steyaert, "A state-space behavioral model for CMOS Class E power amplifiers," IEEE Trans. Computer-Aided Design of Integ. Circuits Systems, vol. 22, no. 2, pp. 132-138, February 2003.

[15] Y. Tanji and H. Kamei, "Behavioral modeling of class-E switching circuits via Weierstrass canonical form," Nonlinear Theory and Its Applications, IEICE, vol. 8, no. 1, pp. 38-48, 2017.

[16] M.K. Kazimierczuk and D. Czarkowski, Resonant Power Converters, John Willy \& Sons, Inc., 2011.

[17] Y. Tanji, "A Newton-type algorithm for determining passive elements including in class-E amplifiers," Proc. NOLTA'12, pp. 628-631, October 2012.

[18] N.O. Sokal, "Class-E RF power amplifiers," QEX, no. 204, pp. 9-20, January/February 2001.

[19] F.Y. Edgeworth, Mathematical Psychics, P. Keagan, London, 1881.

[20] J.A. Nelder and R. Mead, "A simplex method for function minimization," Computer J., vol. 7, pp. 308-313, 1965.

[21] R.E. Massara, Optimization Method in Electronic Circuit Design, John Wiley \& Sons, New York, 1991. 